\title{
Vertically aligned tin-doped indium oxide nanowire arrays: Epitaxial growth and electron field emission properties
}

\author{
Q. $\operatorname{Wan}^{\mathrm{a})}$ \\ Micro-Nano Technologies Research Center, Hunan University, Changsha 410082, \\ People's Republic of China and Department of Electrical Engineering and Computer Science, \\ The University of Michigan, 1301 Beal Avenue, Ann Arbor, Michigan 48109-2122 \\ P. Feng and T. H. Wang \\ Micro-Nano Technologies Research Center, Hunan University, Changsha 410082, \\ People's Republic of China
}

(Received 18 April 2006; accepted 5 July 2006; published online 18 September 2006)

\begin{abstract}
Vertically aligned tin-doped indium oxide (ITO) single-crystalline nanowire arrays are epitaxially grown on ITO/yttrium stabilized zirconia substrates by vapor transport method. Vacuum electron field emission properties of the aligned ITO nanowires are investigated. The turn-on electrical field at a current density of $1 \mu \mathrm{A} / \mathrm{cm}^{2}$ is about $2.0 \mathrm{~V} / \mu \mathrm{m}$, and the lowest vacuum for an obvious emission is $1 \times 10^{-1} \mathrm{~Pa}$. The good performance of field emission is attributed to the vertically aligned morphology, which has a stronger local electric field due to their orientation parallel to the electric-field direction. (C) 2006 American Institute of Physics. [DOI: 10.1063/1.2345278]
\end{abstract}

Tin-doped indium oxide (ITO), an important transparent metal-oxide semiconductor, has been intensively investigated due to its applications in photovoltaics, smart windows, organic light-emitting diodes, and flat-panel displays. ${ }^{1} \mathrm{Up}$ to now, several research groups have reported the growth of ITO nanowires/nanorods based on vapor-liquid-solid growth mode, but no well-aligned ITO nanowire arrays were obtained. $^{2-4}$ Only recently, Wan et al. reported the epitaxial growth of vertically aligned ITO nanowires on latticematched (100) yttrium stabilized zirconia (YSZ) substrates by a thermal evaporation method. ${ }^{5}$

One-dimensional (1D) semiconducting oxide nanostructures were found to be a group of very promising vacuum electron emitters due to their high aspect ratio and small tip radius of curvature. Recently, electron field emission from 1D $\mathrm{ZnO}$ nanostructures, such as nanotetrapods, 6,7 nanowires, ${ }^{8,9}$ nanoneedles, ${ }^{10}$ and nanopins, ${ }^{11}$ has attracted much attention. More recently, the electron field emission properties of other oxide $1 \mathrm{D}$ nanostructures, such as $\mathrm{MoO}_{3}$ nanowires/nanobelts, ${ }^{12,13} \quad \mathrm{IrO}_{2}$ nanowires, ${ }^{14} \quad \mathrm{RuO}_{2}$ nanowires, ${ }^{15} \mathrm{CuO}$ nanowires/nanobelts, ${ }^{16,17}$ and $\mathrm{In}_{2} \mathrm{O}_{3}$ nanowires, ${ }^{18}$ have also been investigated. ITO nanowires should also be a promising candidate as field emitters because of their inherent properties of very low resistivity, thermally stable, and oxidation resistant. In this letter, we report the epitaxial growth and vacuum electron field emission properties of vertically aligned ITO single-crystalline nanowire arrays on ITO/YSZ substrates by vapor transport method.

Figure 1(a) shows the schematic view of our processes for vertically aligned ITO nanowire array growth. First, an ITO (In:Sn=95:5) buffer layer of $200 \mathrm{~nm}$ in thickness was deposited on (100) YSZ single-crystalline substrates (1 $\times 1 \mathrm{~cm}^{2}$ ) by pulsed laser deposition at $600{ }^{\circ} \mathrm{C}$ in the ambient of $2 \times 10^{-2}$ Torr oxygen. A KrF excimer laser $(\lambda=248 \mathrm{~nm}$, pulse duration $=20 \mathrm{~ns}$, repetition frequency $=5 \mathrm{~Hz}$, and pho-

\footnotetext{
a) Author to whom correspondence should be addressed; electronic mail
} wanqing7686@hotmail.com ton energy density $=2 \mathrm{~J} / \mathrm{cm}^{2}$ pulse) was used as the laser source. ITO films grown by pulsed laser deposition method were single-crystalline with a cube-on-cube epitaxial relationship with the YSZ substrate. Subsequently, a gold film with the thickness of about $10 \mathrm{~nm}$ was deposited on ITO/ YSZ substrates by sputter deposition. At last, vertically aligned ITO nanowires were grown by a vapor transport method based on the vapor-liquid-solid (VLS) growth mode.
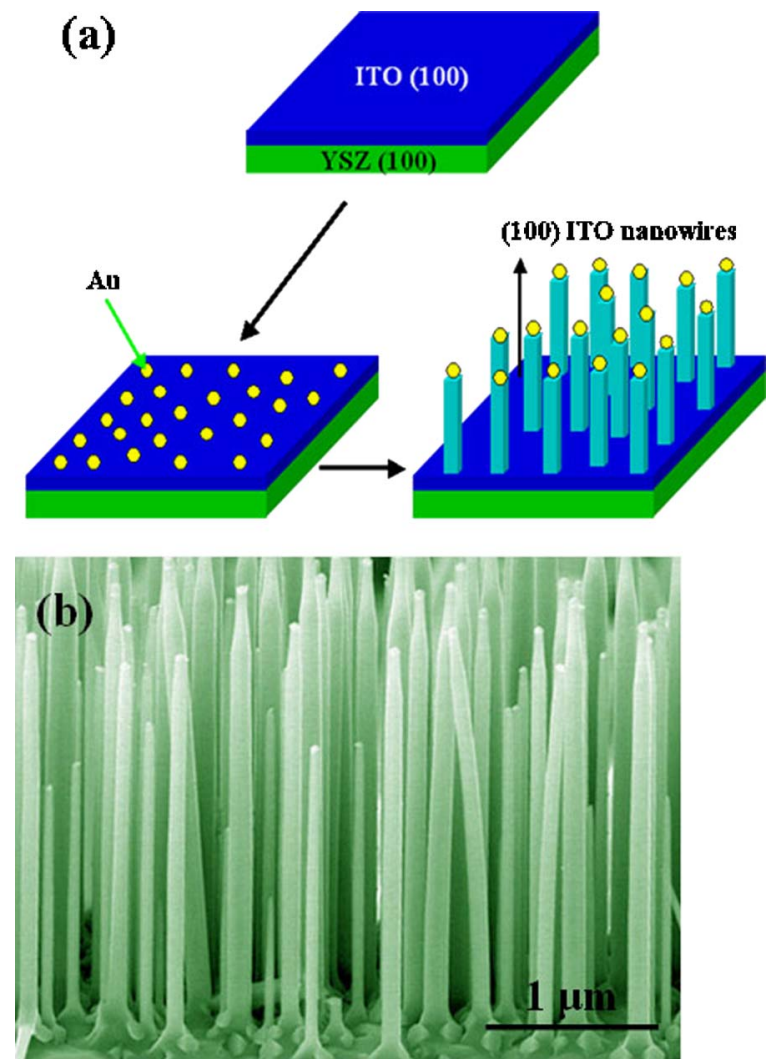

FIG. 1. (Color online) (a) Schematic view of processes for vertically aligned ITO nanowire arrays grown on ITO buffer layer/(100) YSZ substrate. (b) A side-view SEM image of the vertically aligned ITO nanowire arrays grown on ITO/(100) YSZ substrate. 
In the processes of ITO nanowire growth, first, high pure purity (99.99\%) metal indium (In) and stannous oxide ( $\mathrm{SnO})$ powders were mixed thoroughly in the atom ratio of 9:1. The mixture was then loaded in an alumina boat, and an Aucovered ITO/YSZ substrate was also placed on the top of the boat. Then, the alumina boat was positioned at the center of an alumina tube that was inserted into a horizontal tube furnace. The growth temperature was $900{ }^{\circ} \mathrm{C}$ and the growth lasted $1 \mathrm{~h}$ under the $1 \mathrm{l} / \mathrm{min}$ flux of nitrogen with a trace of oxygen.

Figure 1(b) shows a side-view SEM image of the assynthesized ITO nanowires on ITO/YSZ substrate. Vertically aligned ITO nanowire arrays with the mean diameter less than $200 \mathrm{~nm}$ and length of about $2.5 \mu \mathrm{m}$ were obtained. We found that the diameter of the ITO nanowires become smaller near the tip, which is of great significance for field emission application. A nanoparticle is clearly observed at the end of each ITO nanowire, suggesting that the ITO nanowires were grown in the VLS mode. VLS growth mechanism was first proposed by Wagner and Ellis in $1964 .{ }^{19}$ In our experimental case of growing ITO nanowires, VLS process is initiated by the formation of liquid alloy droplet, which contains both Au catalyst and source metals of In and Sn. Precipitation occurs when the liquid droplet becomes supersaturated with the source metals. Under the flow of trace oxygen, ITO nanowire crystal is formed. When (100) ITO buffer layer/YSZ was used as substrate, ITO nanowires grow along (100) crystallographic orientation due to very good epitaxial relationship, leading to vertically aligned ITO nanowire arrays of high crystalline quality. Our experiment also demonstrated that only randomly distributed ITO nanowires were grown on (100) silicon substrate because there is no appropriate epitaxial relationship between (100) Si and ITO.

$\mathrm{X}$-ray pole figure was measured using Schulz reflection method in a two-axis texture goniometry equipped in the same diffractometer. Figure 2(a) shows the (211) pole figure of the ITO nanowire arrays. Fourfold symmetrical $\{112\}$ peaks were observed, which indicates that all ITO nanowires have a cube-on-cube epitaxial relationship with the ITO/YSZ substrate. Transmission electron microscopy (TEM) characterizations were carried out using a JEOL 4000EX-II microscopes operated at $400 \mathrm{kV}$ with a high point-to-point resolution of $0.17 \mathrm{~nm}$ (information limit $0.135 \mathrm{~nm}$ ). Figure 2(b) is a low-magnification bright field TEM image of a single ITO nanowire grown on ITO buffer layer. The inset in Fig. 2(b) is a [100] zone high resolution TEM image, which shows that the wire indeed grows along the [001] direction.

All field emission measurements were carried out in a vacuum chamber with the cathode-anode distance of $200 \mu \mathrm{m}$ at room temperature. The anode is transparent conducting ITO film on the glass, so visible light can be observed when electrons impact the anode. Two curves in Fig. 3(a) illustrate the field emission current density as a function of the applied field in semilogarithmic scale for two vertically aligned ITO nanowire array samples, respectively. Based on the data in Fig. 3(a), a turn-on electrical field of about $2.0 \mathrm{~V} / \mu \mathrm{m}$ was obtained for vertically aligned ITO nanowire arrays at a current density of $1 \mu \mathrm{A} / \mathrm{cm}^{2}$. Vertically aligned ITO nanowires have a stronger local electric field due to their orientation parallel to the electric-field direction. At the same time, vertically aligned ITO nanowires possess more emitter tips, and the tips have better field emission performance than the bodies. Thus, vertically aligned ITO nanowire arrays show better
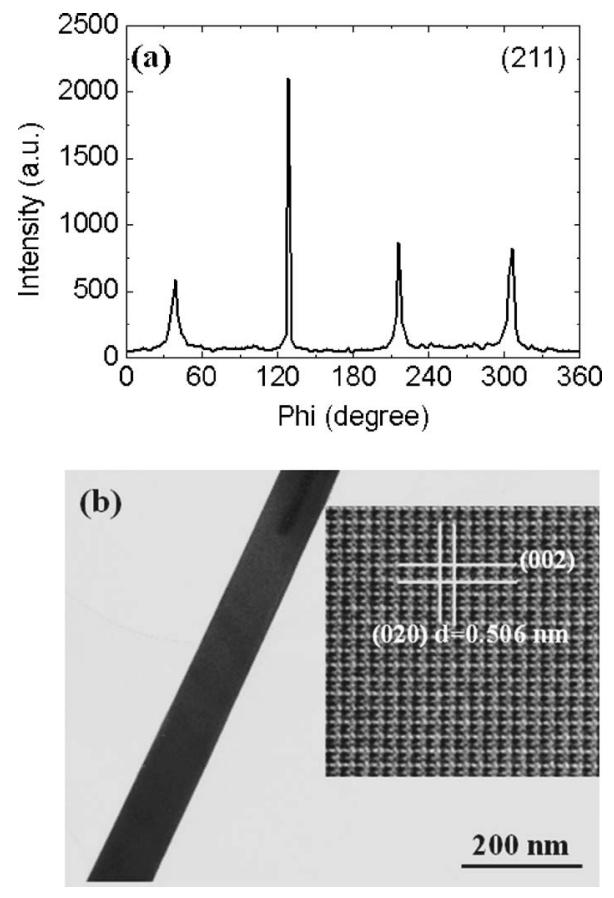

FIG. 2. (a) (211) pole figure of the ITO nanowire arrays on ITO/YSZ substrate. (b) Bright field TEM image of single ITO NWs. The inset is a [100] zone high resolution TEM image, which shows that the growth direction of ITO nanowires is [100].

field emission properties than randomly distributed ITO nanowires.

At room temperature, the field emission current is mostly due to the tunneling of electrons through the surface barrier, which is described by the Fowler-Nordheim (FN) theory. ${ }^{20}$ According to the FN theory, the field emission current $J$ can be expressed in the following form:
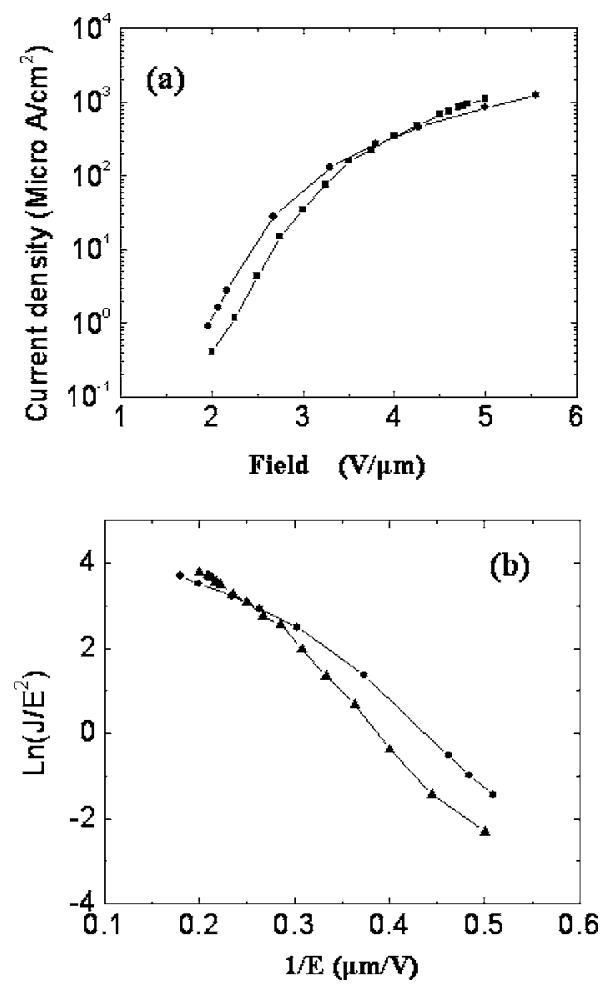

FIG. 3. (a) Field emission current density-field curves of the ITO nanowire arrays measured at the vacuum of $1 \times 10^{-5} \mathrm{~Pa}$. (b) Corresponding FN plots. 

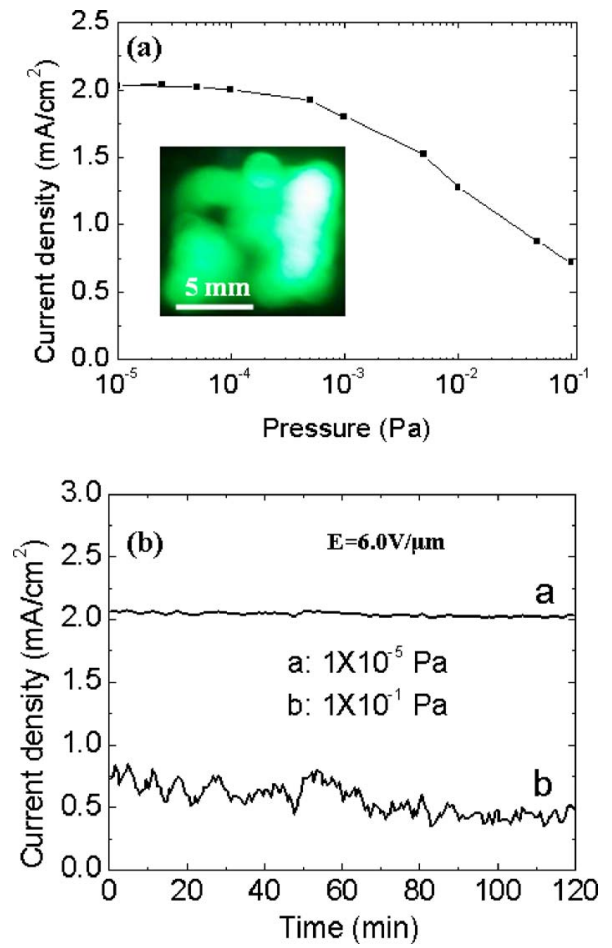

FIG. 4. (Color online) (a) Dependence of emission current density of vertically aligned ITO nanowire arrays with the vacuum pressure in the range from $1 \times 10^{-5}$ to $1 \times 10^{-1} \mathrm{~Pa}$. The inset shows the spatial distribution of emission of ITO nanowires at the $1 \times 10^{-5} \mathrm{~Pa}$. (b) Field emission current stability of ITO nanowire arrays at $1 \times 10^{-5}$ and $1 \times 10^{-1} \mathrm{~Pa}$, respectively.

$$
J=A\left(\beta^{2} E^{2} / \Phi\right) \exp \left(-B \Phi^{3 / 2} / \beta E\right),
$$

where $A=1.4 \times 10^{-6} \exp \left(10.4 / \Phi^{1 / 2}\right)$ in units of $e \mathrm{~A} \mathrm{~V}^{-1}, B$ $=6.8 \times 10^{7}$ in units of $(\mathrm{eV})^{-3 / 2} \mathrm{~V} \mathrm{~cm}^{-1}, \beta$ is the field enhancement factor, $E$ is the applied field $(E=V / d)$, and $\Phi$ is the work function of the emission tip. The exponential dependence between the emission current density and the applied field, as shown in $\ln \left(J / E^{2}\right)-1 / E$ relationship in Fig. 3(b), indicates that the field emission from both ITO nanowire arrays follows the FN relationship. From the slope of the FN curve, we can evaluate the value of $\beta$ ultimately if we know the work function of the emission tip. Adopting the work function of ITO $(\Phi=4.3 \mathrm{eV})$, the $\beta$ of vertically aligned ITO nanowire arrays was estimated to be bout 3850 .

The field emission current dependence with vacuum pressure of the vertically aligned ITO nanowire arrays was studied because environmental stability is one of the main requirements for practical application. As shown in Fig. 4(a), when the vacuum level is higher than $2 \times 10^{-4} \mathrm{~Pa}$, a steady emission current density of about $2.0 \mathrm{~mA} / \mathrm{cm}^{2}$ was measured at the applied field of $6.0 \mathrm{~V} / \mu \mathrm{m}$. The emission current drops slowly in the vacuum range of $5 \times 10^{-4}-1 \times 10^{-1} \mathrm{~Pa}$, and the current density under $1 \times 10^{-1} \mathrm{~Pa}$ is about one-third of the current density at $1 \times 10^{-5} \mathrm{~Pa}$. A field emission recovery was observed when ITO nanowire arrays were operated under the initial pressure of $1 \times 10^{-5} \mathrm{~Pa}$. These results indicated that ITO nanowires show relatively high environmental stability due to their high oxidation resistance. The uniformity of emission from the vertically aligned ITO nanowire arrays was examined at the field of $6.0 \mathrm{~V} / \mu \mathrm{m}$ using the transparent anode. As shown by the inset image in Fig. 4(a), the emission is very bright and relatively homogeneous. The field emission stability of vertically aligned ITO nanowire arrays was investigated by measuring the current fluctuation with time at a fixed electric field of $6.0 \mathrm{~V} / \mu \mathrm{m}$. As shown in Fig. $4(\mathrm{~b})$, during $2 \mathrm{~h}$ of continuous operation at $1 \times 10^{-5} \mathrm{~Pa}$, no obvious degradation of current density was found and the current fluctuation was as low as $\pm 5 \%$, but the current fluctuation becomes larger when vacuum reduced, and we found that the current fluctuation was $\pm 28 \%$ at $1 \times 10^{-1} \mathrm{~Pa}$.

In summary, vertically aligned ITO nanowire arrays were epitaxially grown on (100) YSZ substrates with ITO buffer layer by vapor transport method. Vertically aligned ITO nanowire arrays showed an enhanced field emission with a turn-on field of about $2.0 \mathrm{~V} / \mu \mathrm{m}$ at a current density of $1 \mu \mathrm{A} / \mathrm{cm}^{2}$. Our results demonstrated that vertically aligned ITO nanowire arrays were very promising for vacuum electron field emission applications.

This work was partly supported by the Chinese Ministry of Education (Grant No. 705040), the Special Funds for Major State Basic Research Project (No. G2001CB3095), and the National Natural Science Foundation of China (Nos. 60236010).

${ }^{1}$ N. Taga, H. Odaka, Y. Shigesato, I. Yasui, M. Kamei, and T. E. Haynes, J. Appl. Phys. 80, 978 (1996).

${ }^{2}$ Q. Wan, Z. T. Zong, S. L. Feng, and T. H. Wang, Appl. Phys. Lett. 85, 4759 (2004).

${ }^{3}$ S. Y. Li, C. Y. Lee, P. Lin, and T. Y. Tseng, Nanotechnology 16, 451 (2005).

${ }^{4}$ Y. Q. Chen, J. Jiang, B. Wang, and J. G. Hou, J. Phys. D 37, 3319 (2004).

${ }^{5}$ Q. Wan, M. Wei, D. Zhi, J. L. MacManus-Driscoll, and M. G. Blamire, Adv. Mater. (Weinheim, Ger.) 18, 234 (2006).

${ }^{6}$ Q. Wan, K. Yu, T. H. Wang, and C. L. Lin, Appl. Phys. Lett. 83, 2253 (2003).

${ }^{7}$ Q. H. Li, Q. Wan, Y. J. Chen, T. H. Wang, H. B. Jia, and D. P. Yu, Appl. Phys. Lett. 85, 636 (2004).

${ }^{8}$ L. F. Dong, J. Jiao, D. W. Tuggle, J. M. Petty, S. A. Elliff, and M. Coulter, Appl. Phys. Lett. 82, 1096 (2003).

${ }^{9}$ C. J. Lee, T. J. Lee, S. C. Lyu, Y. Zhang, H. Ruh, and H. J. Lee, Appl. Phys. Lett. 81, 3648 (2002).

${ }^{10}$ Y. W. Zhu, H. Z. Zhang, X. C. Sun, S. Q. Feng, J. Xu, Q. Zhao, B. Xiang, R. M. Wang, and D. P. Yu, Appl. Phys. Lett. 83, 144 (2003).

${ }^{11}$ C. X. Xu and X. W. Sun, Appl. Phys. Lett. 83, 3806 (2003).

${ }^{12}$ J. Zhou, S. Z. Deng, N. S. Xu, J. Chen, and J. C. She, Appl. Phys. Lett. 83, 2653 (2003).

${ }^{13}$ Y. B. Li, Y. Bando, D. Golberg, and K. Kurashima, Appl. Phys. Lett. 81, 5048 (2002).

${ }^{14}$ R. S. Chen, Y. S. Huang, Y. M. Liang, C. S. Hsieh, D. S. Tsai, and K. K. Tiong, Appl. Phys. Lett. 84, 1552 (2004).

${ }^{15}$ C. S. Hsieh, D. S. Tsai, R. S. Chen, and Y. S. Huang, Appl. Phys. Lett. 85, 3860 (2004).

${ }^{16}$ Chien-Te Hsieh, Jin-Ming Chen, Hung-Hsiao Lin, and Han-Chang Shih, Appl. Phys. Lett. 83, 3383 (2003).

${ }^{17}$ J. Chen, S. Z. Deng, N. S. Xu, W. Zhang, X. Wen, and S. Yang, Appl. Phys. Lett. 83, 746 (2003).

${ }^{18}$ S. Q. Li, Y. X. Liang, and T. H. Wang, Appl. Phys. Lett. 87, 43104 (2005).

${ }^{19}$ R. S. Wagner and W. C. Ellis, Appl. Phys. Lett. 4, 89 (1964).

${ }^{20}$ R. H. Fowler and L. W. Nordheim, Proc. R. Soc. London, Ser. A 119, 173 (1928). 